\title{
Debris studies for high-repetition rate and high-power laser experiments at the Central Laser Facility
}

\author{
N. Booth, S. Astbury, E. Bryce, R.J. Clarke, C.D. Gregory, \\ J.S. Green, D. Haddock, R.I. Heathcote and C. Spindloe
}

\section{Published version information:}

Citation: N Booth et al. "Debris studies for high-repetition rate and high-power laser experiments at the Central Laser Facility." Proceeding of SPIE, vol. 10763 (2018): 107630S. Is in proceedings of: Radiation Detectors in Medicine, Industry, and National Security XIX, San Diego, California, USA, 19-23 Aug 2018.

DOI: $\underline{10.1117 / 12.2318946}$

Copyright 2018 Society of Photo-Optical Instrumentation Engineers (SPIE). One print or electronic copy may be made for personal use only. Systematic reproduction and distribution, duplication of any material in this publication for a fee or for commercial purposes, and modification of the contents of the publication are prohibited.

This version is made available in accordance with publisher policies. Please cite only the published version using the reference above. This is the citation assigned by the publisher at the time of issuing the APV. Please check the publisher's website for any updates. 


\section{Debris studies for high-repetition rate and high-power laser experiments at the Central Laser Facility}

Booth, N., Astbury, S., Bryce, E., Clarke, R., Gregory, C., et al.

N. Booth, S. Astbury, E. Bryce, R. J. Clarke, C. D. Gregory, J. S. Green, D. Haddock, R. I. Heathcote, C. Spindloe, "Debris studies for high-repetition rate and high-power laser experiments at the Central Laser Facility," Proc. SPIE 10763, Radiation Detectors in Medicine, Industry, and National Security XIX, 107630S (11 September 2018); doi: 10.1117/12.2318946

SPIE Event: SPIE Optical Engineering + Applications, 2018, San Diego, California, United States 


\title{
Debris studies for high-repetition rate and high-power laser experiments at the Central Laser Facility
}

\author{
N. Booth*, S. Astbury, E. Bryce, R.J. Clarke, C.D. Gregory, J.S. Green, \\ D. Haddock, R.I. Heathcote and C. Spindloe \\ Central Laser Facility, STFC Rutherford Appleton Laboratory, Harwell Campus, Didcot, UK
}

\begin{abstract}
Many of the new large European facilities that are in the process of coming online will be operating at high power and high repetition rates. The ability to operate at high repetition rates is important for studies including secondary source generation and inertial confinement fusion research. In these interaction conditions, with solid targets, debris mitigation for the protection of beamline and diagnostic equipment becomes of the upmost importance. These facilities have the potential to take hundreds, if not thousands, of shots every day, creating massive volumes of debris and shot materials.

In recent testing of the Central Laser Facility's High Accuracy Microtargetry Supply (HAMS) system on the midrepetition rate Gemini facility ( $15 \mathrm{~J}, 40 \mathrm{fs}, 1$ shot every 20 seconds), diagnostics were deployed in order to specifically look at the debris emitted from targets designed for high repetition rate experiments. By using a high frame rate camera, it has been possible to observe and characterize some of the debris production, whilst also looking at target fratricide.

Alongside these results from Gemini, we also present results of static debris measurements undertaken on the Vulcan Petawatt high energy, high power facility, where the cumulative effects of debris produced by high power laser experiments have been observed.

Keywords: Debris, High Power Lasers, High Repetition Rate Lasers, Central Laser Facility, Vulcan Laser, Gemini Laser

*nicola.booth@stfc.ac.uk
\end{abstract}

\section{INTRODUCTION}

The Central Laser Facility (CLF) has been embarking on a project to enable delivery of large numbers of complex targetry to user experiments, in increasing volumes, for higher repetition rate operations. This comprehensive project incorporates all aspects of delivery to the users, from target fabrication techniques, to mechatronic stages, to how to position the targets accurately at the focus of the laser[1]. The Gemini facility at the CLF has been operating for the past ten years at a shot rate of one shot every 40 seconds, which scales the volume of targets required for a full experiment from approximately 100 on the Vulcan facility, to the order of $\sim 1000$ 's. Unlike other facilities that operate high repetition facilities with large single foil targets or plasma mirror disks with long focal lengths[2][3], the goal of the CLF's project is to have individual targets, to be positioned at the interaction point from an $f / 2$ focusing parabola at 0.1 $\mathrm{Hz}$ or faster.

Alongside the issue of delivering targets at these high shot rates; solid target experiments generate large volumes of debris, which is of great concern to facilities which will be offering experiments at $\mathrm{Hz}$ to even $\mathrm{kHz}$ shot rates at Petawatt, and even greater, powers. This debris can be ejected from targets as high velocity projectiles, which is often the cause of damage to expensive optical elements in the interaction chamber. Through collaborative experiments on both the Gemini and Vulcan Petawatt facilities between the target fabrication group and the experimental scientists at the CLF, we have been able to observe debris production under a variety of laser/target configurations to ascertain information on how we might best limit damage to the facility, whilst delivering our experimental program at ever increasing repetition rates.

Radiation Detectors in Medicine, Industry, and National Security XIX, edited by Gary P. Grim,

Lars R. Furenlid, H. Bradford Barber, Proc. of SPIE Vol. 10763, 107630S · @ 2018 SPIE

CCC code: $0277-786 \mathrm{X} / 18 / \$ 18 \cdot$ doi: $10.1117 / 12.2318946$ 


\section{GEMINI HIGH REPETITION RATE EXPERIMENTS}

In order to be able to operate experiments in such harsh conditions, we embarked on experiments to observe target fratricide, how such effects may be reduced, what kind of debris and projectiles are produced during a high power laser shot, and how we might look to mitigate both the volume of debris and the impact it can have on the surrounding optics and equipment. Previous observations in the Vulcan Petawatt target chamber have shown large chunks of debris of the order of a few $\mathrm{mm}$ in size can be found embedded in the chamber walls, and obviously this would have catastrophic consequences to the experiment if these were impacting into the focussing parabola. The Gemini facility is a different environment to Vulcan, operating at much lower energies $(\sim 10 \mathrm{~J}$ rather than $\sim 500 \mathrm{~J})$ but operates at higher repetition rates than Vulcan (one shot every 40 seconds rather than 1 shot per 20 minutes) with similar intensities on target. Anecdotal evidence from Gemini has shown that thin film pellicles that are used to protect the parabolas from damage may survive intact for the full duration of an experiment, or may fail at random points during experiments, and very little is known about the mechanisms which cause them to fail.

Experiments on the Gemini facility which primarily shoot gas targets have routinely been firing shots at 1 shot every 20 seconds, with $f / 40$ and $f / 20$ focussing options. The aim of the current set of experiments was to see if we could increase the shot rate for solid target experiments to somewhere close to that, considering the shorter focal length optic $(f / 2)$ and the requirement to position the target within the Raleigh range of this optic reliably ( $\pm 4 \mu \mathrm{m}$ to be in the centre of this range). We also wanted to look at what problems we might encounter from target fratricide and the risks that are placed on the surrounding optics from target debris.

\subsection{High Accuracy Microtargetry Supply system}

The High Accuracy Microtargetry Supply (HAMS) system that the CLF are developing for high repetition rate solid target experiments consists of 4 separate components. The mechatronic stages consist of xz linear stages, a tripod which performs y, tip and tilt, and a rotation stage. There is then a stable interface wheel which has been designed and characterized to have a flatness of $2 \mu \mathrm{m}$ across its surface. The targets are manufactured using MEMS techniques[4], which allows for batch production, and are formed of membranes supported over silicon wafer segments, of which up to 8 can be mounted on each interface wheel, allowing in the order of hundreds of targets on each wheel. The targets are then aligned to the focus of the Gemini laser using a multi-wavelength interferometer. The components of the HAMS system are shown in Figure 1.

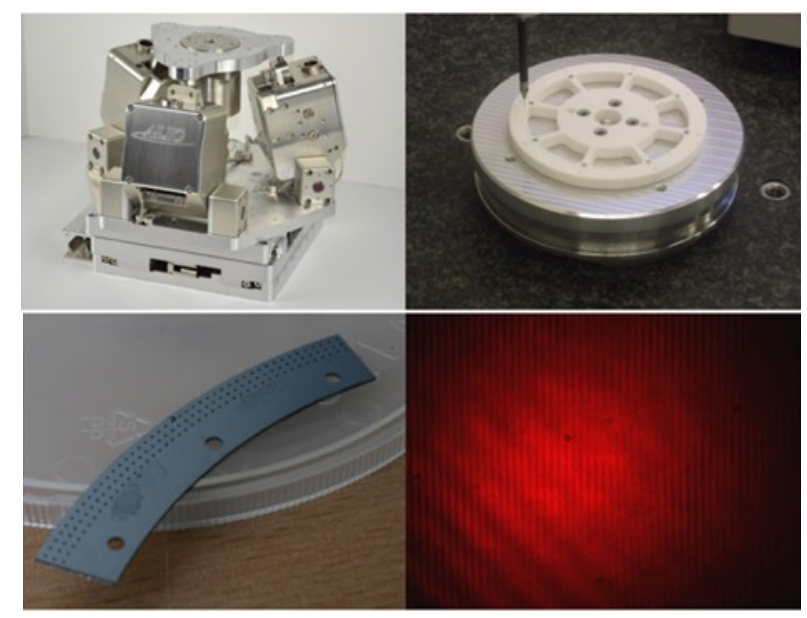

Figure 1: The components of the HAMS system, incorporating the 6 axis stage (rotation not shown), the interface wheel, the target sections and the alignment system. 


\subsection{Experiment Details}

The aim of the experiment was to test the components of the project together in an experiment situation, whilst also looking particularly at the survivability of targets in different geometries.

The experiments were performed using the Gemini laser system at the CLF. One beamline of Gemini was used, with laser pulses containing approximately $15 \mathrm{~J}$ post compression, along with the Gemini double plasma mirror system[5] to enhance the laser contrast, which gives an $\sim 65 \%$ throughput of energy. After the plasma mirrors we have approximately $10 \mathrm{~J}$ in $45 \mathrm{fs}$ on target, at $800 \mathrm{~nm}$. The beam is focussed with an $f / 2$ parabola and focuses to a best spot of $\approx 2.5 \mu \mathrm{m}$ diameter with intensities on target of $\approx 10^{21} \mathrm{Wcm}^{-2}$.

The main diagnostics for the experiment were a probe beam reflected from the rear surface of the target to look at the plasma expansion[6], and a high frame rate camera[7] to look at the debris projectiles from the target. The high frame rate camera was back illuminated with a CW laser, and the experimental geometry is shown below in Figure 2.

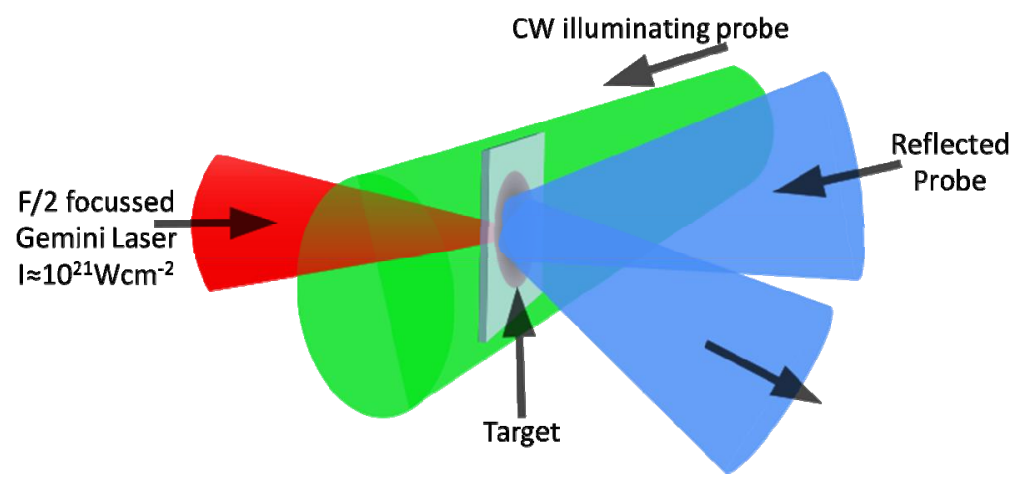

Figure 2: Sketch of the experimental layout, showing the Gemini $\mathrm{f} / 2$ focusing beam, the reflected rear surface probe, and the $\mathrm{CW}$ probe beam for the high frame rate camera illumination.

\subsection{Experiment Targetry}

The main targets that were used in the experiment were $100 \mathrm{~nm}$ thickness Silicon Nitride foils suspended over apertures in a silicon wafer. We then tested different geometries of these targets, both circular and square apertures with varying diameters, and different mounting. The center to center distances for each of the target types remain the same, therefore the edge to edge separation is varied. We also tested some $1 \mu \mathrm{m}$ thickness parylene targets with $2 \times 2 \mathrm{~mm}^{2}$ windows in a full silicon wheel. Both of these target types are shown in Figure 3. By shooting these different target geometries, we aim to discover if there was a particular point in the target geometry which meant that on shot, targets surrounding the shot target would survive better or alternatively be destroyed easier.

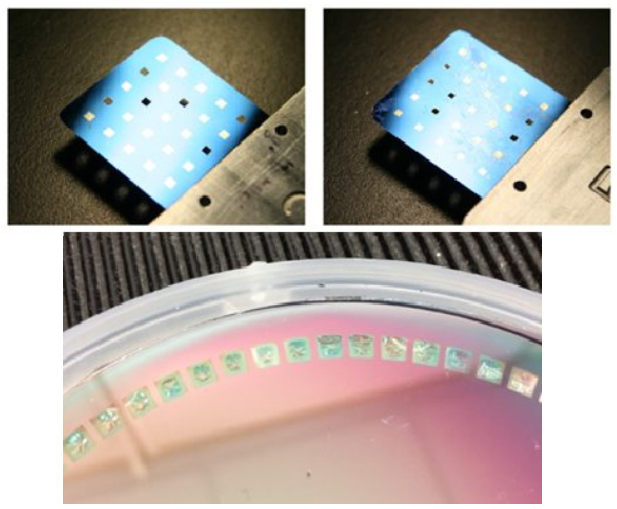

Figure 3: Some of the different target types tested in this experiment, including 'standard' array targets with variations in the hole geometries, and a silicon wafer mount. 
We shot a number of the targets of each type, making measurements of the volume and the velocity of debris produced directly from each target, and recording observations of target damage in the surrounding targets, to correlate the two occurrences.

\subsection{Debris Projectile Measurements}

A high frame rate camera operating at 100000 or 210000 frames per second was used to capture videos of the laser-target interaction. The target was back-illuminated by a $532 \mathrm{~nm} \mathrm{CW}$ laser, and videos were captured over $10 \mathrm{~ms}$.

Figure 4 is an image from the rear surface probe and shows one of the complex targets that were shot in this experiment, a silicon nitride target suspended on a $300 \times 300 \mu \mathrm{m}^{2}$ silicon frame, in a $1 \times 1 \mathrm{~mm}^{2}$ hole. In Figure 5 a sequence of still images from this shot captured by the high frame rate camera is shown. Here we have a number of time steps, showing the evolution of debris produced in the interaction. From Figure 4 we can see that the laser shot actually clipped the silicon frame, and in the initial $10 \mu \mathrm{s}$ after the interaction, a lot of small pieces of debris are ejected by the plasma formed on both the front and rear of the target, at subsequent times, larger pieces of debris are ejected more slowly from both the front and rear of the targets. As far out as $450 \mu \mathrm{s}$ after the interaction, a large number of large pieces of debris are still being ejected from the target.

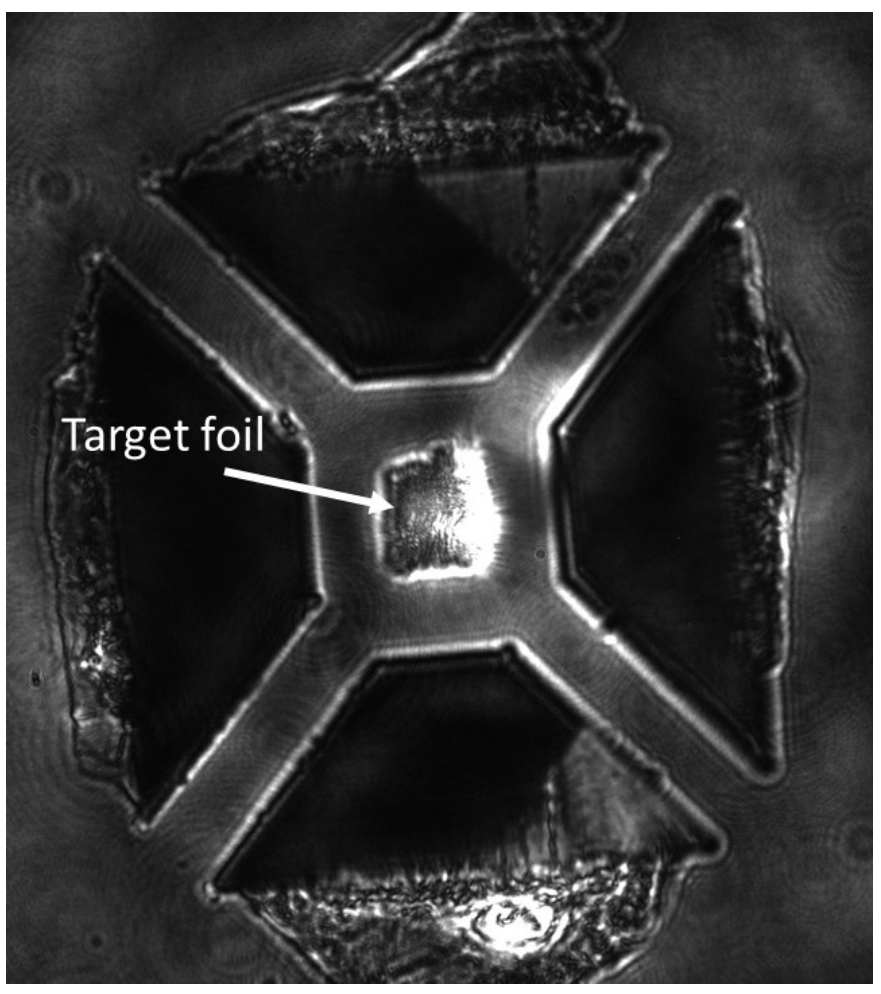

Figure 4: On shot rear surface probe data showing the laser clipping the target frame of a 100nm thick silicon nitride target suspended on a silicon frame of $300 \times 300 \mu \mathrm{m}^{2}$. 


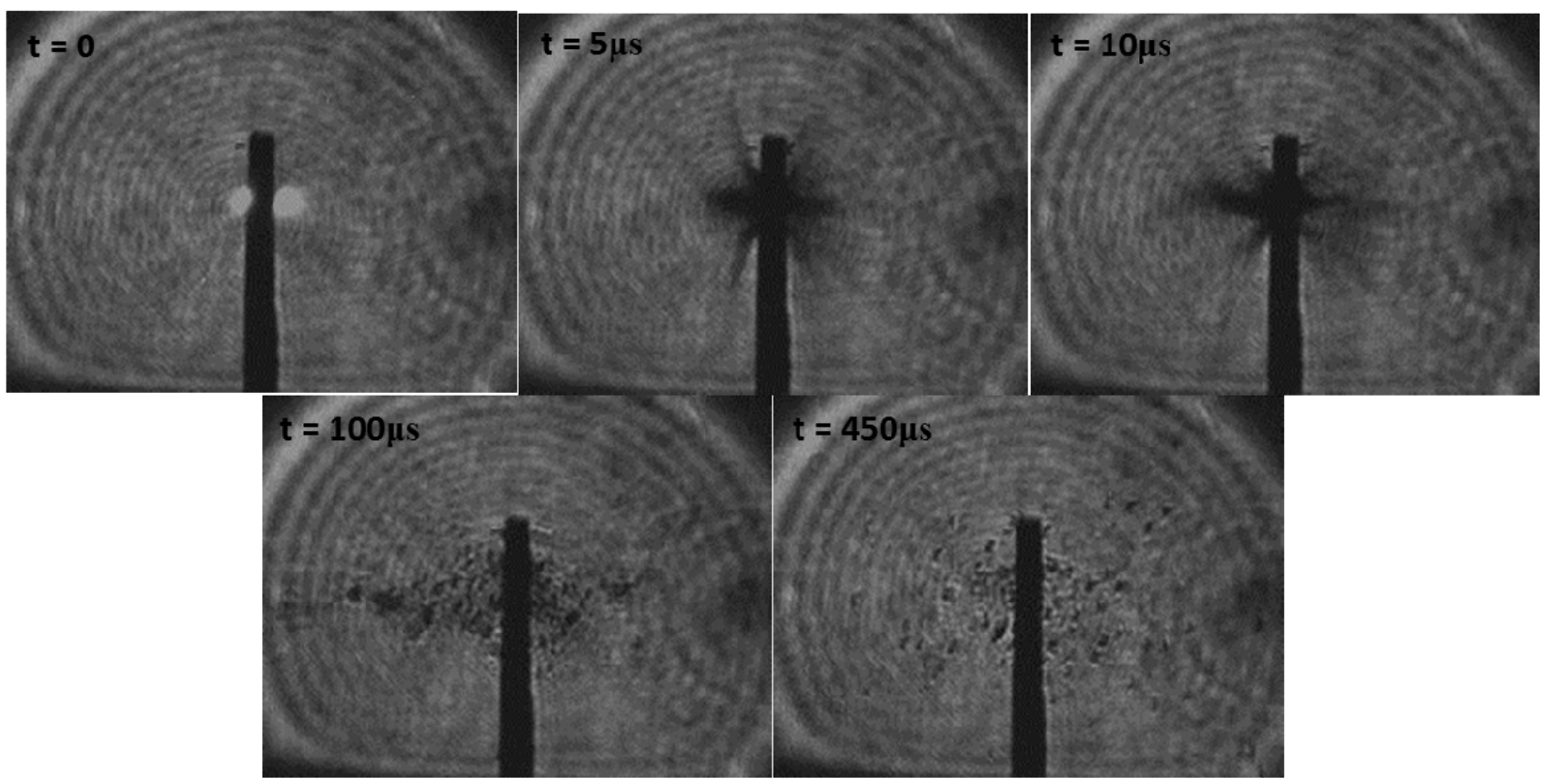

Figure 5: High frame rate camera images $(210 \mathrm{kfps})$ at $\mathrm{t}=0,5,10,100$ and $450 \mu \mathrm{s}$ from the arrival of the laser pulse on a silicon nitride target with a $300 \mu \mathrm{m}$ diameter aperture. At $\mathrm{t}=0$ the initial plasma flash at the interaction point can be seen.

The ejection of projectiles from the target (and the mount) has been analyzed to look at the speed with which they are ejected. The histogram of the data shown in Figure 6, shows that the majority of the particles are slow travelling at under $2 \mathrm{~ms}^{-1}$, with a relatively small distribution of other particles at speeds between 2 and $60 \mathrm{~ms}^{-1}$, with another spike in the distribution at speeds greater than $60 \mathrm{~ms}^{-1}$, which are the particles ejected by the initial interaction and plasma formation on the target surface. Some of the fastest debris emitted from the interaction are at speeds around $100 \mathrm{~ms}^{-1}$. Since we can see in Figure 4 that we hit the silicon 'spokes' with the laser, a lot of the debris produced in this shot was from the silicon supports.

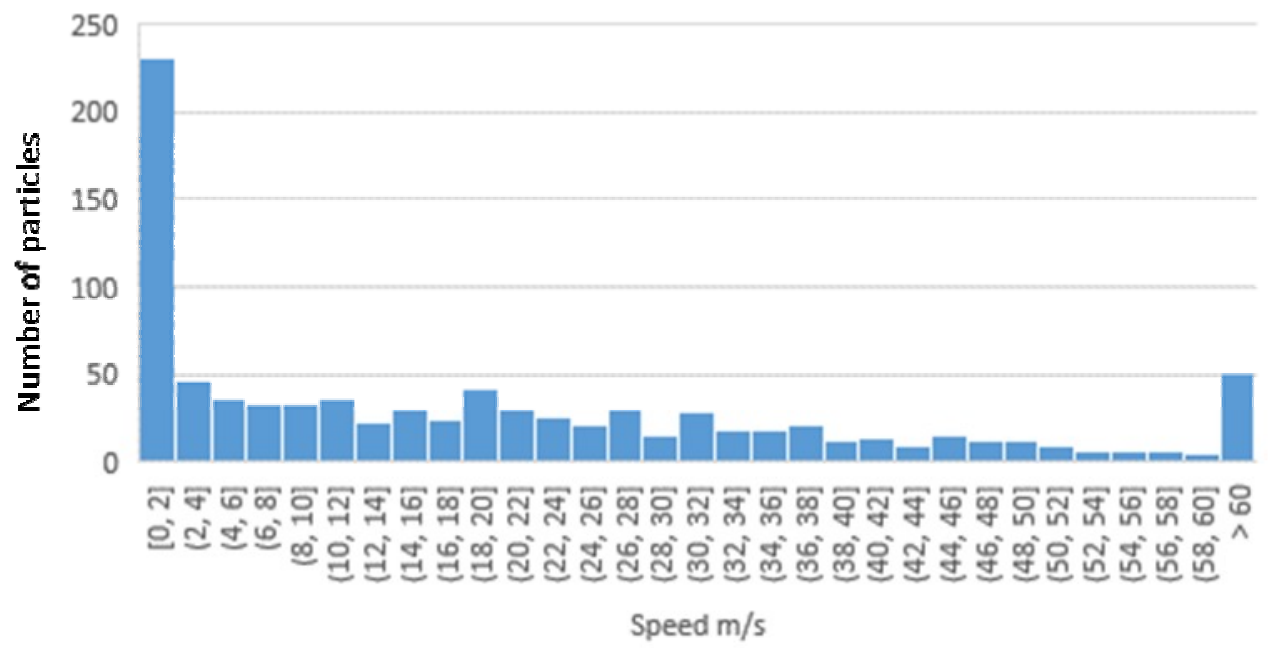

Figure 6: Histogram of the speed of projectiles ejected from the target shot in Figure 5 . The majority of debris is ejected at under $2 \mathrm{~ms}^{-1}$, whereas a peak can also be seen in very fast projectiles at $>60 \mathrm{~ms}^{-1}$.

In Figure 7 we have high frame rate camera images from a silicon nitride target in a $2 \mathrm{~mm}$ diameter aperture. In this shot we hit the center of the target, with no damage created to the silicon mount. Here we can see that the projectiles ejected 
by the initial laser-plasma interaction are in the direction back towards the laser, whilst there are fewer particles in both directions at later times.

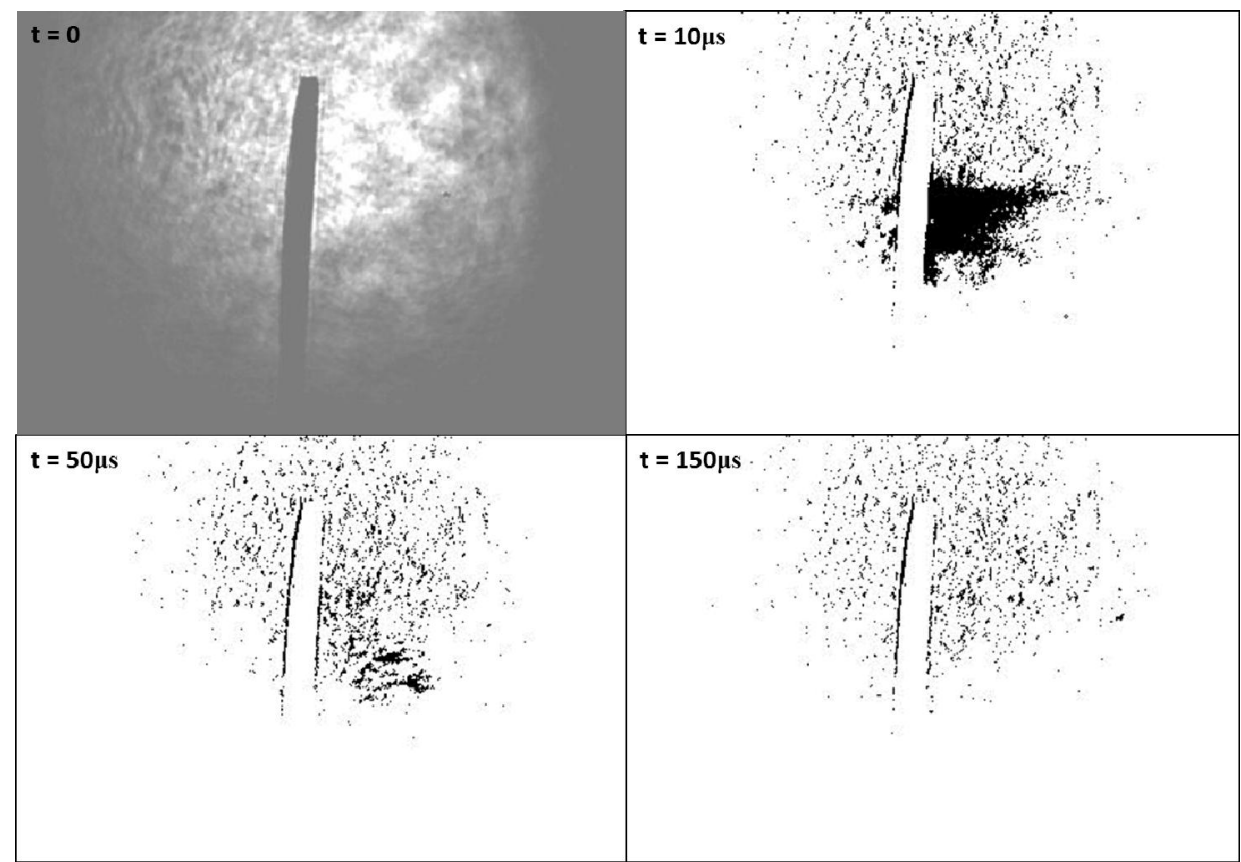

Figure 7: High frame rate camera images with background subtracted at $t=0,10,50$ and $150 \mu$ s from the arrival of the laser pulse on a $2 \mathrm{~mm}$ diameter silicon nitride target. In this shot, we see that the majority of the debris is ejected from the front of the target. The image is background subtracted, and a large amount of noise can be seen in the images.

From this target where we have no damage to the mount, there is a much smaller quantity of particles of all speeds ejected from the target, as seen in the histogram in Figure 8. We also see a much more even distribution of speeds of particles ejected after the initial plasma ejection. The analysis program is limited by particle size and is not sophisticated enough to pick out the individual particles ejected after the initial interaction. Clearly from the $10 \mu$ s image in Figure 7 there are a large number of particles ejected in a large mass at very fast speeds (above $40 \mathrm{~ms}^{-1}$ ) which are not identified in the histogram in Figure 8.

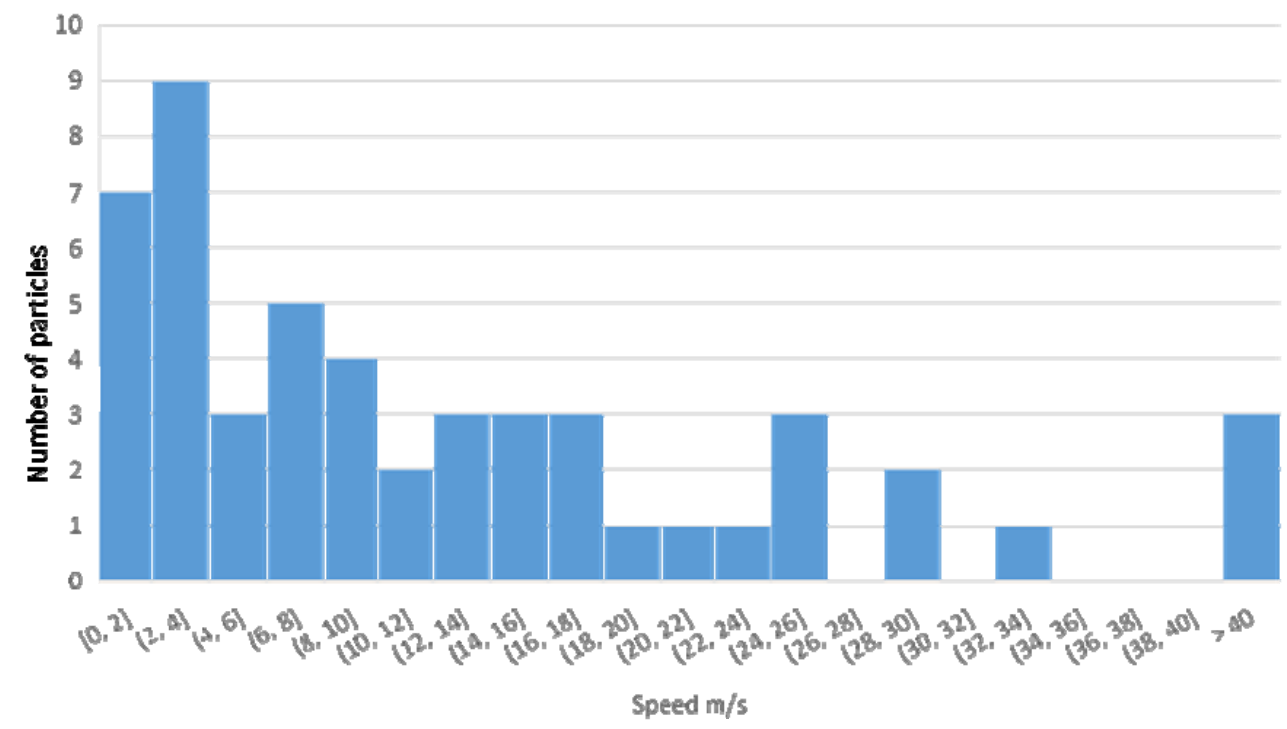

Figure 8: Histogram of the speed of projectiles ejected from the target shot in Figure 7. 


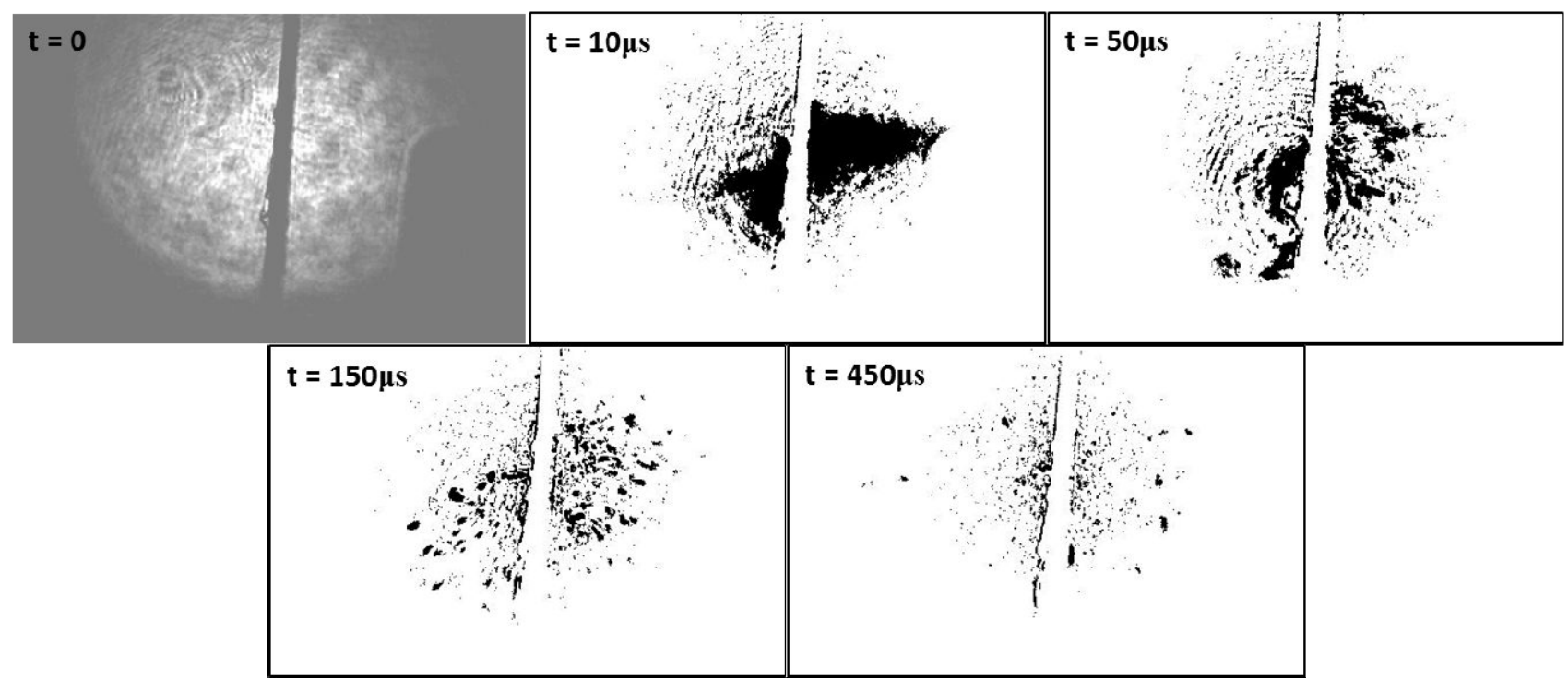

Figure 9: High frame rate camera images with background subtracted at $\mathrm{t}=0,10,50,150$ and $450 \mu$ s from the arrival of the laser pulse on a parylene target with a $2 \mathrm{~mm}$ square aperture. In this shot, the laser was slightly misaligned and the silicon target mount was clipped by the laser, and so much larger pieces of debris are seen at longer timescales after the interaction.

The laser shot in Figure 9 was slightly misaligned, and the laser clipped some of the target mount in the interaction. At later times, much larger pieces of debris can be seen. The Gemini $f / 2$ parabola is protected by a nitrocellulose thin film pellicle, which prevents both small and large pieces of debris from impacting into the parabola. In this instance some of the large pieces of debris travelling back towards the parabola from the shattered target mount broke the protective pellicle (an example of a broken pellicle is shown in Figure 10). Without the pellicle to protect the parabola, the debris would have coated the optic and potentially damaged both the coating and the substrate.

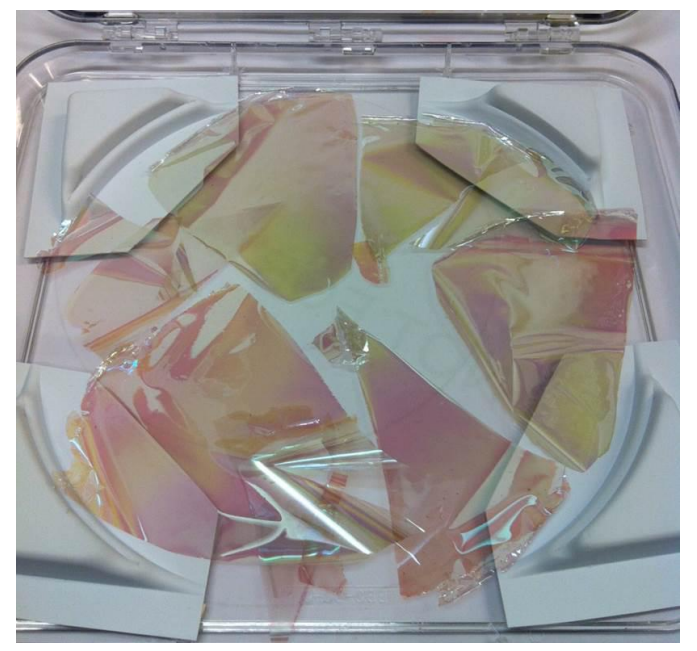

Figure 10: Gemini thin film pellicle designed to protect the parabola from impacts. In this instance the pellicle was broken by a large piece of target debris, which was successfully prevented from impacting the parabola.

The volume of debris seen in these experiments is variable, and depends greatly on the size of target and alignment of the laser. The silicon mounts are very brittle, and misalignment of the laser can cause catastrophic failure of the target, and potentially very damaging consequences for the surrounding optics. 


\section{VULCAN HIGH ENERGY EXPERIMENTS}

The Vulcan target areas of the CLF operate high energy experiments, in the Petawatt area delivering $600 \mathrm{~J}$ in $600 \mathrm{fs}$, and in Target area West (TAW) delivering 2 short pulse beams with up a maximum of $300 \mathrm{~J}$ in $2-15 \mathrm{ps}$, and 6 long pulse beamlines with up to $280 \mathrm{~J}$ in $6 \mathrm{~ns}$ with shots on target every 20 minutes.

In order to look at the impact of debris on the focusing parabolas in these target areas, we exposed three different standard coating samples to debris in the TAW chamber. These three coatings were dielectric, protected silver and protected gold. In the Petawatt target area, we have routinely been monitoring the debris produced in experiments with a series of debris witness plates placed around the chamber, where we also observe that, as with Gemini, the majority of debris is in the direction back towards the parabola.

One half of the substrate was covered by a $1 \mathrm{~mm}$ thick aluminum plate to give a control surface. In Figure 11, we have images of the substrates after exposure to the debris from a TAW experiment. The silver coating quite clearly shows the most damage, removing almost the entirety of the protective coating from the optic. Both the gold and dielectric coatings sustained a reasonable amount of damage. After exposure to the debris, we rotated the aluminum plate to expose the lower half of the mirror to the laser, to observe how the damaged coating is then affected by the laser footprint.

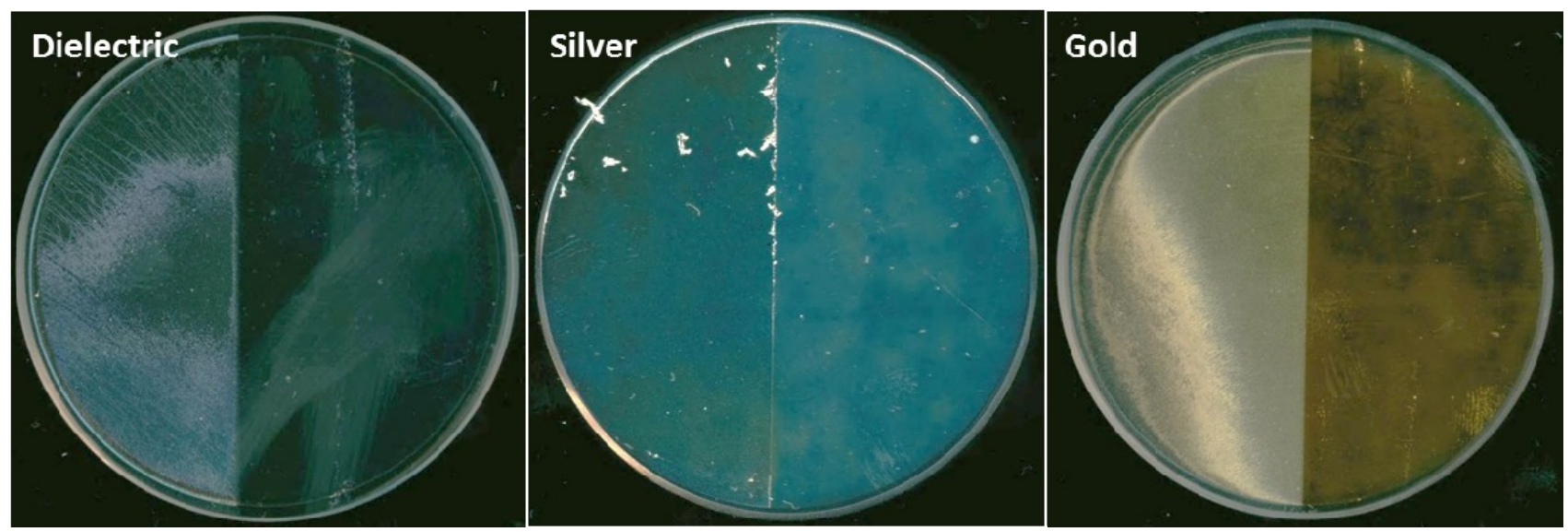

Figure 11: Debris exposure tests for dielectric, silver and gold coated mirrors. The protected silver coating shows lots of damage and lifting of the coating, whilst the gold shows some evaporation of the coating. The dielectric coating shows some signs of stress, but does not overly impact on the performance of the mirror.

In figure 12, we can see that the bottom left hand quadrant which was exposed to both the debris and the laser has sustained a large about of damage, and has in fact removed the reflective surface from the mirror. The gold and dielectric substrates fared better in this test, with the dielectric coating showing the least damage to the reflectivity of the surface.

Across both the Vulcan and Gemini target areas, the final focusing optics are silver coated parabolas. Dielectric coatings are often not available for the range of angles that our shorter focal length parabolas operate with, and silver coatings also have an advantage in that they are reasonably simple and cost effective to strip and recoat, rather than requiring the substrate surface to be repolished. As we know we have significant debris issues in our interaction chambers, we have continued with silver coatings. However the $\mathrm{f} / 3$ parabola in the Petawatt interaction chamber has recently been replaced with a dielectric coated optic, as the number of impact sites on the old substrate meant that the coating was degrading to a point where it was needed to be recoated after only a few experiments reducing the cost effectiveness of that choice. 


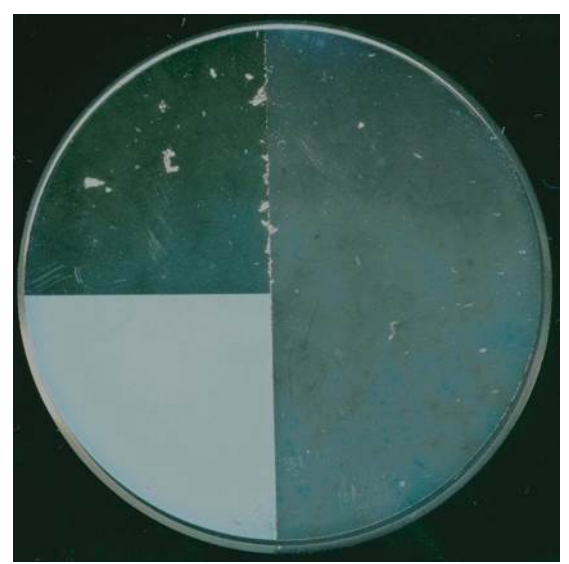

Figure 12: Silver mirror after debris exposure then laser exposure showing reflective coating removal in the segment of the mirror exposed to both debris and laser.

\section{CONCLUSIONS}

Debris production in high power laser experiments has the potential to have very costly and damaging consequences for experiments. The Gemini experiments have demonstrated the importance of laser-target alignment in minimizing debris production as we move towards experiments operating at high repetition rates of $1 \mathrm{~Hz}$ or higher. Unfortunately we see that the majority of the debris from the target is ejected from the front surface of the target, and the Vulcan experiments demonstrate the need to protect the parabolas with thin film or glass pellicles.

\section{REFERENCES}

[1] N. Booth, R. Clarke, R. Heathcote, D. Neely, R. Pattathil, D. Rusby, C. Spindloe, D. Symes, M. Tolley, and S. Tomlinson, "High-rep rate target development for ultra-intense interaction science at the Central Laser Facility," 2014, p. 921107.

[2] C. Serbanescu, S. Fourmaux, J. C. Kieffer, R. E. Kincaid, and a Krol, “A K-alpha x-ray source using high energy and high repetion rate laser system for phase contrast imaging," Soc Photo Opt Instrum Eng, pp. 1-12, 2009.

[3] A. Borot, D. Douillet, G. Iaquaniello, T. Lefrou, P. Audebert, J. Geindre, A. Borot, D. Douillet, G. Iaquaniello, T. Lefrou, P. Audebert, and J. Geindre, "High repetition rate plasma mirror device for attosecond science," vol. 13104, no. 2014, 2016.

[4] C. Spindloe, G. Arthur, F. Hall, S. Tomlinson, R. Potter, S. Kar, J. Green, A. Higginbotham, N. Booth, and M. K. Tolley, "High volume fabrication of laser targets using MEMS techniques," J. Phys. Conf. Ser., vol. 713, no. 1, 2016.

[5] M. J. V Streeter, P. S. Foster, F. H. Cameron, R. Bickerton, S. Blake, P. Brummit, B. Costello, E. Divall, C. Hooker, P. Holligan, D. R. Neville, P. P. Rajeev, D. Rose, J. Suarez-Merchen, D. Neely, D. C. Carroll, L. Romagnani, and M. Borghesi, "Astra Gemini compact plasma mirror system," Cent. Laser Facil. Annu. Rep., 2008.

[6] J. S. Green, C. D. Murphy, N. Booth, R. J. Dance, R. J. Gray, D. A. Maclellan, P. McKenna, D. Rusby, and L. Wilson, "Single shot, temporally and spatially resolved measurements of fast electron dynamics using a chirped optical probe," J. Instrum., vol. 9, no. 3, 2014.

"https://photron.com/fastcam-sa-z/." 